\title{
Inverse Variational Problem for Nonlinear Dynamical Systems
}

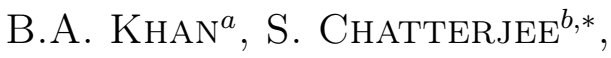 \\ S.G. $\mathrm{ALI}^{c}$ AND B. TALUKDAR ${ }^{d}$ \\ ${ }^{a}$ Department of Physics, Krishnath College, Berhampore, Murshidabad 742101, India \\ ${ }^{b}$ Department of Physics, Bidhannagar College, EB-2, Sector-1, Salt Lake, Kolkata 700064, India \\ ${ }^{c}$ Department of Physics, Kazi Nazrul University, Asansol 713303, India \\ ${ }^{d}$ Department of Physics, Visva-Bharati University, Santiniketan 731235, India
}

Received: 23.09.2021 \& Accepted: 10.12.2021

Doi: $10.12693 /$ APhysPolA.141.64

*e-mail: supriya_2k1@rediffmail.com

\begin{abstract}
Two different approaches to solving the inverse problem of the calculus of variation for nonlinear equations are introduced. The first approach is based on an integral representation of the Lagrangian function, while the second one relies on the generalization of Lagrangian symmetry. As an application of the first approach, we initially provide some useful remarks on the Lagrangians of the modified Emden-type equation, and then construct Lagrangian functions for (i) a cubic-quintic Duffing oscillator, (ii) Liénardtype oscillator and (iii) Mathews-Lakshmanan oscillator. Using the second approach, we obtain analytic (Lagrangian) representations for the three velocity-dependent equations, namely, (iv) Abraham-Lorentz oscillator, (v) Helmholtz oscillator and (vi) Van der Pol oscillator. For each of the systems in (i)-(vi) we find the Jacobi integral and thereby provide a method for obtaining the Hamiltonian function.
\end{abstract}

topics: Lagrangians, Jacobi integrals, Hamiltonians, nonlinear differential equations

\section{Introduction}

The inverse problem in the calculus of variation involves deciding whether a given system of second-order ordinary differential equations representing dynamical systems is a solution of the Euler-Lagrange equation and eventually finding its Lagrangian representation, if the solution exists [1]. For linear ordinary differential equations, the set of constraints for the existence of Lagrangians is provided by the so-called Helmholtz conditions $[2,3]$. The equation of motion of a damped harmonic oscillator

$$
\ddot{x}(t)+\gamma \dot{x}(t)+\omega^{2} x(t)=0
$$

violates these conditions such that we cannot find a time-independent Lagrangian representation for it. In (1), the over-dots denote the differentiation with respect to $t$. Here $\gamma$ represents the frictional coefficient of the medium in which the oscillator of angular frequency $\omega$ is embedded. An explicitly time-dependent Lagrangian of the damped system was actually found $[4,5]$ during 1940's. For this Lagrangian, the canonical momentum is time-dependent. This provides an awkward analytical constraint to use the corresponding Hamiltonian to quantize the system [6]. In 1931, Bateman $[7,8]$ suggested a very ingenious method to find an explicitly time-independent Lagrangian for the damped harmonic oscillator by doubling the number of the system's degrees of freedom. More specifically, in conjunction with (1), an auxiliary oscillator equation

$$
\ddot{y}(t)-\gamma \dot{y}(t)+\omega^{2} y(t)=0
$$

was considered to obtain the Lagrangian

$$
\begin{aligned}
L & =\dot{x}(t) \dot{y}(t)+\frac{\gamma}{2}(x(t) \dot{y}(t)-\dot{x}(t) y(t)) \\
& -\omega^{2} x(t) y(t) .
\end{aligned}
$$

Physically, the energy drained out from the oscillator in (1) is completely absorbed by that in (2) such that these two oscillators together represent a conservative system. The Euler-Lagrange equation [9] written in terms of $y(t)(x(t))$ gives the equation of motion for $x(t)(y(t))$. Because of this unusual behavior, the Lagrangian (3) is said to provide an indirect analytic (Lagrangian) representation of the system. The canonical quantization of damped harmonic oscillator using the indirect Lagrangian representation has been found to be quite satisfactory [10-12] because the corresponding Hamiltonian is time independent [5].

Traditionally, the Lagrangian function $L$ of the autonomous differential equation is expressed as $L=T-V$, where $T$ and $V$ stand respectively for the kinetic and potential energies of the system represented by the equation. Such a Lagrangian is referred to as standard. Relatively recently, a new type of Lagrangian functions [13] has been proposed 
for dissipative-like autonomous differential equations. These do involve neither $T$ nor $V$. As a result, such Lagrangians were qualified as non-standard. One can also find non-standard Lagrangian representation for linear differential equations which violate Helmholtz conditions [14]. At the end of the last decade, Musielak [15] and Cieśliński and Nikiciuk [16] presented methods to write results for non-standard Lagrangians of variety of nonlinear dynamical systems. It was observed that some of the equations follow from the inverse type of Lagrangian functions, while others follow logarithmic functions. For example, the modified Emden-type equation [17]

$$
\ddot{x}+\alpha x \dot{x}+\beta x^{3}=0, \quad x=x(t),
$$

was found to have Lagrangian representations given by [13]

$$
\begin{aligned}
& \qquad L=\frac{1}{\dot{x}+k x^{2}} \quad \text { for } \quad \alpha=3 k \text { and } \beta=\frac{\alpha^{2}}{9} \text { (5) } \\
& \text { and } \\
& \qquad L=\ln \left(\dot{x}+k x^{2}\right) \quad \text { for } \quad \alpha=4 k \text { and } \beta=\frac{\alpha^{2}}{8} .
\end{aligned}
$$

Writing (4)-(6) we omitted the argument $t$ from $x(t)$. We shall follow this convention throughout. The results for Lagrangians in (4) and similar equations were chosen in an ad hoc fashion rather than being derived from the solution of the inverse problem in the calculus of variation [18].

In this context, we note that the work of Nucci and Tamizhmani [19] is a step forward along this line of investigation because the authors made the use of the elegant method due to Jacobi [20] to find the Lagrangian functions of some differential equations (linear or nonlinear). The purpose of the present paper is to introduce two uncomplicated methods to solve the inverse variational problem for nonlinear differential equations, and then use them to construct non-standard Lagrangian representations for a number of evolution equations that play a role in many applicative contexts. The first method of our interest makes use of the integral representation of the Lagrangian function in terms of the first integral of the equation of motion [21]. The second method [22] sought by us depends on a simple but nontrivial generalization of the Lagrangian symmetry [23].

In Sect. 2, we provide a brief derivation of the methods of our interest to solve the inverse problem. As a useful application of the first method, we demonstrate that the Lagrangian in (4) can be found for arbitrary values of $\alpha$ and $\beta$ such that the results in (5) and (6) are only special instances. Further, we show that for $\alpha=3 k$ and $\beta=\alpha^{2} / 9$ one can have a logarithmic Lagrangian representation of (4) in addition to the inverse one as given in (5). But for $\alpha=4 k$ and $\beta=\alpha^{2} / 8$, (4) does not have any inverse type Lagrangian. Using the second method we provide an ab intio derivation of the Bateman Lagrangian in (3) and find that the approach is especially important for velocitydependent nonlinear differential equations. We devote Sect. 3 to present an uncomplicated method of finding the first integral of nonlinear differential equations and thereby obtain Lagrangian representations of a number dynamical systems using our first method. Where-ever possible, we present results for both inverse and logarithmic Lagrangians types. Here, we also implement the second method to find Lagrangians for two nonlinear dissipative equations and one third-order linear equation. In Sect. 4, we look for Jacobi integrals [9] for the nonstandard Lagrangians found in Sect. 3, and then provide results for the corresponding Hamiltonian functions. And finally, in Sect. 5 we summarize our outlook on the present work and make some concluding remarks.

\section{Methods for constructing Lagrangian representation}

\subsection{Method 1: Lagrangian function from an integral representation}

The relationship between the Lagrangian and constant of the motion is represented by the Jacobi integral [9]

$$
\sum_{i=1}^{N} v^{i}\left(\frac{\partial L}{\partial v^{i}}\right)-L=K
$$

where $L=L(\boldsymbol{x}, \boldsymbol{v})$ is the Lagrangian and $K=$ $K(\boldsymbol{x}, \boldsymbol{v})$ is the constant of the motion of the secondorder ordinary differential equation

$$
\ddot{x}^{i}=f^{i}\left(x^{j}, \dot{x}^{j}\right), \quad i=1,2, \ldots, N .
$$

Here $\boldsymbol{x}=\left(x^{1}, \ldots, x^{N}\right)$ and $\boldsymbol{v}=\dot{\boldsymbol{x}}=\left(v^{1}, \ldots, v^{N}\right)$. The equations for the characteristics of (7) are [24]

$$
\frac{\mathrm{d} v^{1}}{v^{1}}=\ldots=\frac{\mathrm{d} v^{N}}{v^{N}}=\frac{\mathrm{d} L}{L+K}
$$

Writing (8) in the equivalent form $\frac{\mathrm{d} v^{i}}{\mathrm{~d} t}=f^{i}(\boldsymbol{x}, \boldsymbol{v})$, one can demand that the constant of the motion $K(\boldsymbol{x}, \boldsymbol{v})$ is the first integral of the equation provided

$$
\sum_{i=1}^{N}\left(f^{i}(\boldsymbol{x}, \boldsymbol{v}) \frac{\partial K}{\partial v^{i}}+v^{i} \frac{\partial K}{\partial x^{i}}\right)=0 .
$$

The solution of (10) or the integral surfaces can now be obtained from

$$
\frac{\mathrm{d} v^{1}}{f^{1}(\boldsymbol{x}, \boldsymbol{v})}=\ldots=\frac{\mathrm{d} v^{N}}{f^{N}(\boldsymbol{x}, \boldsymbol{v})}=\frac{\mathrm{d} x^{1}}{v^{1}}=\ldots=\frac{\mathrm{d} x^{N}}{v^{N}} \text {. }
$$

For an $N$-dimensional autonomous Newtonian system the solution of (7) can be expressed as an integral over possible constants of the motion [21], and thus we have

$$
L(\boldsymbol{x}, \boldsymbol{v})=\frac{1}{N} \sum_{i=1}^{N} v^{i} \int^{v^{i}} \mathrm{~d} \xi \frac{K^{i}(\boldsymbol{x}, \xi)}{\xi^{2}} .
$$

For the one-dimensional system (12) reads

$$
L(x, v)=v \int^{v} \mathrm{~d} \xi \frac{K(x, \xi)}{\xi^{2}} .
$$


In the following we shall use the integral representation of the Lagrangian function in (13) to deal with the inverse problem of (4) in a more general context. To find the constant of the motion, we shall follow a very simple prescription, rather than taking recourse to the use of (10) and (11).

We begin by writing (4) in the autonomous form

$$
v(x) v^{\prime}(x)+\alpha x v(x)+\beta x^{3}=0,
$$

where $v(x)=\frac{\mathrm{d} x}{\mathrm{~d} t}$ and $v^{\prime}(x)=\frac{\mathrm{d} v(x)}{\mathrm{d} x}$. This first-order linear differential equation can be solved to get the constant of the motion

$$
\begin{aligned}
& K(x, \dot{x})=\frac{2 \alpha}{\sqrt{\alpha^{2}-8 \beta}} \arctan \left(\frac{h\left(\alpha x^{2}+4 \dot{x}\right)}{x^{2} \sqrt{\alpha^{2}-8 \beta}}\right) \\
& \quad+\ln \left(\beta x^{4}+\alpha x^{2} \dot{x}+2 x^{2}\right) .
\end{aligned}
$$

In principle, (15) can be used in (13) to construct a Lagrangian for a modified Emden-type equation. But it would be instructive to write from (15), a constant of the motion for $\alpha=3 k$ and $\beta=\alpha^{2} / 9$, and find for (5) two Lagrangian representations, which are not related by a trivial gauge term. For these special values of the parameters, the constant of the motion reduces to a very simple form given by

$$
K(x, \dot{x})=\frac{\left(k x^{2}+\dot{x}\right)^{2}}{k x^{2}+2 \dot{x}} .
$$

If $K(x, \dot{x})$ is a constant of the motion, its reciprocal

$$
K_{1}(x, \dot{x})=\frac{1}{K(x, \dot{x})}
$$

is also a constant of the motion. It is straightforward to verify that $K_{1}(x, \dot{x})$ in conjunction with (12) leads to the reciprocal Lagrangian as appears in (5). Similarly, using (16) we obtain a logarithmictype Lagrangian

$$
L_{1}=\frac{1}{2} \dot{x} \ln \left(k x^{2}+2 \dot{x}\right)-k x^{2} .
$$

The Lagrangians (5) and (18) for the same equation of motion are not related by the gauge term. Such Lagrangians are called alternative or inequivalent Lagrangians [25]. The existence of alternative Lagrangian description of physical systems has important consequences for the correspondence between symmetries and constants of motion [26].

\subsection{Method 2: Lagrangian from equation of motion}

While recognizing the importance of the first integral in solving the inverse variational problem, Hojman et al. [27] raised a very important question. Can the equations of motion themselves, rather than their first integrals, be used to provide a Lagrangian description of mechanical systems? In [22], it was firmly established that for linear Newtonian systems one can always use the equations of motion to find a satisfactory solution to the inverse problem.
It is well known in classical mechanics that the so-called Noether's theorem [23] provides a relation between symmetries of the Lagrangian with the conserved quantities of the equation of motion. It is less well known, however, that the symmetries of the equations of motion form a larger set than the symmetries of the Lagrangians. However, if s-equivalence is taken into account, the set of Lagrangian symmetries coincides with that of the equation of motion. By s-equivalence, we mean a Lagrangian symmetry in which several constants of the motion may be associated with one symmetry transformation [28]. Understandably, the study in [27] is a kind of generalization of the traditional Noetherian symmetry. In this context, an interesting result that was found is outlined below.

If the second-order differential equation (8) follows from an action principle with the Lagrangian $L\left(x^{i}, \dot{x}^{i}\right)$, then this equation will also follow from a higher-order action characterized by an acceleration-dependent Lagrangian $\bar{L}\left(x^{i}, \dot{x}^{i}, \ddot{x}^{i}\right)$ given by

$$
\bar{L}\left(x^{i}, \dot{x}^{i}, \ddot{x}^{i}\right)=\mu_{i}\left(\ddot{x}^{i}-f^{i}\left(x^{i}, \dot{x}^{i}\right)\right) .
$$

The Lagrangians $L\left(x^{i}, \dot{x}^{i}\right)$ and $\bar{L}\left(x^{i}, \dot{x}^{i}, \ddot{x}^{i}\right)$ are related by a gauge term written as

$$
L\left(x^{i}, \dot{x}^{i}\right)=\bar{L}\left(x^{i}, \dot{x}^{i}, \ddot{x}^{i}\right)+\frac{\mathrm{d} g\left(x^{i}, \dot{x}^{i}\right)}{\mathrm{d} t}
$$

such that

$$
\mu_{i}=-\frac{\partial g\left(x^{i}, \dot{x}^{i}\right)}{\partial \dot{x}^{i}} .
$$

In general, the concept of higher-order action that leads to a generalized classical mechanics is due to Euler who found the differential equation $[29,30]$

$$
\sum_{i=0}^{n}(-1)^{i}\left(\frac{\mathrm{d}}{\mathrm{d} t}\right)^{i} \frac{\partial L}{\partial x^{(i)}}=0
$$

for the $n$-th order Lagrangian $L=$ $L\left(x^{i}, \dot{x}^{i}, \ddot{x}^{i}, \ldots, x^{(n)}\right)$. We shall now show that the auxiliary equation (2) introduced by Bateman [7] to find the time-independent Lagrangian for a damped harmonic oscillator follows naturally from (19) and thereby obtain the result in (3).

From (1) and (19) we write

$$
\bar{L}(x, \dot{x}, \ddot{x}, y)=y\left(\ddot{x}+\gamma \dot{x}+\omega^{2} x\right)
$$

and substitute (23) in the second-order EulerLagrange equation obtained from (22) for $n=2$. In writing (23), we have identified $\mu_{1}$ as $y$. This gives the expected equation

$$
\ddot{y}-\gamma \dot{y}+\omega^{2} y=0 .
$$

In view of (20), we write the second-order Lagrangian for the uncoupled systems in (1) and (2) as

$$
\begin{aligned}
& L(x, \dot{x}, \ddot{x}, y, \dot{y}, \ddot{y})=y\left(\ddot{x}+\gamma \dot{x}+\omega^{2} x\right) \\
& \quad+x\left(\ddot{y}-\gamma \dot{y}+\omega^{2} y\right)-\frac{\mathrm{d}}{\mathrm{d} t}(y \dot{x}+x \dot{y}) .
\end{aligned}
$$

The third term in (25) stands for the gauge term of the second-order Lagrangian [31]. The above Lagrangian gives (3) to within a multiplicative 
constant. As a useful addendum we will show in Sect. 3 that the method can be adapted to deal with nonlinear problems.

\section{Lagrangians for nonlinear dynamical systems}

Here we first will use Method 1 (Sect. 2.1) to compute results for the Lagrangian representation of (i) a cubic-quintic Duffing oscillator [32], (ii) a Liénard-type nonlinear oscillator or generalized Emden-type equation [17] and (iii) MathewsLakshmanan oscillator [33]. Similar results for dissipative systems (iv) Abraham-Lorentz oscillator [34], (v) Helmholtz oscillator [35] and (vi) Van der Pol oscillator [36] will then be obtained by the use of Method 2 (see Sect.2.2). The oscillator in (iv) is represented by a third-order linear differential equation and its Lagrangian function plays a crucial role in quantizing the radiation-damped harmonic oscillator [37].

(i) The cubic-quintic Duffing oscillator represented by

$$
\ddot{x}+a x+b x^{3}+c x^{5}=0
$$

with constant values of $a, b$ and $c$ arise in a number of applicative contexts [38]. The firstorder autonomous differential equation corresponding to (26) given by

$$
v(x) v^{\prime}(x)+a x+b x^{3}+c x^{5}=0
$$

can easily be integrated to get the constant of the motion

$$
K(x, \dot{x})=6 a x^{2}+3 b x^{4}+2 c x^{6}+6 \dot{x}^{2} .
$$

From (13) and (28) we find the Lagrangian

$$
L=\frac{1}{2} \dot{x}^{2}-\frac{1}{2} a x^{2}-\frac{1}{4} b x^{4}-\frac{1}{6} c x^{6} \text {. }
$$

Since (26) can easily be integrated to get a constant of the motion, the above result for $L$ could also be obtained by using the famous formula $L=T-V$. But a number of inequivalent Lagrangians can be constructed by using various powers of the first integral in (28). Thus in close analogy with our observation on the results of the modified Emden-type equation (4), it may be of some interest to find the Lagrangian of $(26)$ for $K_{1}(x, \dot{x})$. In this case we get a fairly complicated result

$$
L_{1}=\frac{1}{r x^{2}}+\frac{\sqrt{6} \dot{x}}{r^{3 / 2} x^{3}} \arctan \left(\frac{\sqrt{6} \dot{x}}{\sqrt{r} x}\right)
$$

with

$$
r=6 a+3 b x^{2}+2 c x^{4} .
$$

The cubic-quintic Duffing oscillator is primarily represented by the standard Lagrangian (27). But (30) shows that it can also be represented by a non-standard Lagrangian. In an interesting paper Urenda-Cazares et al. [39] obtained the integral of motion for a damped cubic-quintic Duffing oscillator with variable coefficients. In the absence of damping the equation considered in [39] coincides with that of ours as given in (26) except that the coefficients $a, b, c$ are now time-dependent. The constant of the motion for this undamped oscillator can also be found by converting it into the autonomous form and solving the resultant equation. The time dependence of the coefficients poses no problem because $t$ now enters the autonomous equation as a parameter.

In the standard procedure to construct timedependent constant of the motion for damped nonlinear systems, one applies the well-known Lie symmetry method [23, 40]. Urenda-Cazares et al. [39], however, followed a different route to find a first integral for the damped system. In particular, they sought to eliminate the damping term from the equation by using a transformation that is often employed to remove first-order term from a second-order ordinary differential equation. This permitted them to treat the damped and undamped systems on equal footing. We are interested here to find analytic representation of damped systems using the equation of motion only. In the Appendix we present our results for the Lagrangian and corresponding Jacobi integral [9] for the damped cubic-quintic Duffing oscillator and make some appropriate comments.

(ii) Here the equation of our interest is the Liénard-type nonlinear oscillator represented by [17]

$$
\ddot{x}+k x \dot{x}+\lambda x+\frac{k^{2}}{9} x^{3}=0 .
$$

When studying the dynamical properties of (32), Chandrasekar et al. [41] provided a non-standard Lagrangian representation for (32). In respect of this we shall show that a relatively simpler representation can be obtained by using the integral representation (13). For (32), an equation analogous to that in $(27)$ reads

$$
v(x) v^{\prime}(x)+k x v(x)+\lambda x+\frac{k^{2}}{9} x^{3}=0 .
$$

The first integral obtained from (33) can be found as

$$
K(x, \dot{x})=\frac{\left(9 \lambda+3 k \dot{x}+k^{2} x^{2}\right)^{2}}{9 \lambda+6 k \dot{x}+k^{2} x^{2}} .
$$

From (13) we now obtain the Lagrangian

$$
L=3 k \dot{x} \ln \left(9 \lambda+6 k \dot{x}+k^{2} x^{2}\right)-2 k^{2} x^{2} .
$$

Similarly, for $K_{1}(x, \dot{x})=1 / K(x, \dot{x})$ we find

$$
L_{1}=\frac{1}{9 \lambda+3 k \dot{x}+k^{2} x^{2}} .
$$

Interestingly, from (35) and (36) we see that as with (4) (for $\alpha=3 k, \beta=\alpha^{2} / 9$ ) the Liénard-type oscillator can also have both logarithmic and inverse Lagrangian representation.

(iii) The Mathews-Lakshmanan oscillator [33]

$$
\ddot{x}+\frac{\lambda x}{1-\lambda x^{2}} \dot{x}^{2}+\frac{\omega^{2} x}{1-\lambda x^{2}}=0
$$

may be regarded as the zero-dimensional version of the scalar non-polynomial field equation [42]. 
It can also be considered as an oscillator with a position-dependent effective mass [43]. Writing (37) as an autonomous first-order differential equation, we found its first integral as

$$
K(x, \dot{x})=\frac{\lambda \dot{x}^{2}-\omega^{2}}{1+\lambda x^{2}} .
$$

In fact, (38) in conjunction with (13) leads to the well known result for the Lagrangian [44], i.e,

$$
L=\frac{\lambda \dot{x}^{2}+\omega^{2}}{1+\lambda x^{2}}
$$

Similarly, the constant of the motion $K_{1}(x, \dot{x})=1 / K(x, \dot{x})$ gives

$$
L_{1}=\left(1+\lambda x^{2}\right)\left[\omega-\sqrt{\lambda} \dot{x} \arctan \left(h \frac{\sqrt{\lambda} \dot{x}}{\omega}\right)\right] \text {. }
$$

Since $\arctan (h x)=\frac{1}{2} \ln \left(\frac{1+x}{1-x}\right)$, the fairly complicated result in (40) actually represents a logarithmic Lagrangian.

(iv) The motion of an accelerated charged particle including the reactive effects is given by the so-called Abraham-Lorentz equation [34] which in one dimensional case can be written as

$$
m \ddot{x}+k x-m \tau \dddot{x}=0 .
$$

The third term in (41) with

$$
\tau=\frac{2}{3} \frac{e^{2}}{m c^{2}}
$$

has its origin in the radiative reaction and represents the jerk term in the equation of motion. We shall present here an indirect Lagrangian representation of (41). To that end, we write a Lagrangian

$$
\bar{L}=y(m \ddot{x}+k x-m \tau \dddot{x})
$$

characterized by two degrees of freedom, namely, $x(t)$ and $y(t)$. The third-order Lagrangian when substituted in (22) for $n=3$ gives the associated equation [7]

$$
m \ddot{y}+k y+m \tau \dddot{y}=0
$$

such that we can write an indirect Lagrangian

$$
\begin{aligned}
L & =y(m \ddot{x}+k x-m \tau \dddot{x}) \\
& +x(m \ddot{y}+k y+m \tau \dddot{y})+a-b
\end{aligned}
$$

for the dual system. Here, $a$ and $b$ given by

$$
a=m \tau \frac{\mathrm{d}}{\mathrm{d} t}(y \ddot{x}-x \ddot{y})
$$

and

$$
b=m \frac{\mathrm{d}}{\mathrm{d} t}(y \dot{x}+x \dot{y})
$$

represent the appropriate gauge terms which have been used to write (45) in the maximally reduced form

$$
L=m \dot{x} \dot{y}+\frac{m \tau}{2}(\dot{x} \ddot{y}-\dot{y} \ddot{x})-k x y .
$$

From (48) we see that we need a second-order Lagrangian to analytically represent the third-order equation (41). As expected in the absence of radiative reaction, i.e., $\tau=0$, (48) gives the usual harmonic oscillator Lagrangian. (v) The simple nonlinear differential equation [45]

$$
\ddot{x}+\delta \dot{x}+\alpha x-\beta x^{2}=0
$$

with constant values $\alpha, \beta$ and $\delta$ represent the Helmholtz oscillator. Since it involves a dissipative term linear in velocity, its Lagrangian representation can be found following the method used in the previous example. But here we should remember that linear and nonlinear terms contribute to a Lagrangian with unequal weights [46]. The weight factors are determined by demanding that the computed Lagrangian function should reproduce the equation of motion via the Euler-Lagrange equation. Thus, in close analogy with (43), we write

$$
\bar{L}=y\left(\ddot{x}+\delta \dot{x}+\alpha x-w \beta x^{2}\right),
$$

where $w$ stands for the required weight factor. In writing (50) we assumed that weight factor of the linear terms is unity. The postulated second-order Lagrangian in (50) leads to the associated equation

$$
\ddot{y}-\delta \dot{y}+\alpha y-2 w \beta x y=0
$$

that we need to construct $L$. We thus write the Lagrangian for (49) in the form

$$
\begin{aligned}
L & =-\frac{\mathrm{d}}{\mathrm{d} t}(y \dot{x}+\dot{y} x)+y\left(\ddot{x}+\delta \dot{x}+\alpha x-w \beta x^{2}\right) \\
& +x(\ddot{y}-\delta \dot{y}+\alpha y-2 w \beta x y) .
\end{aligned}
$$

We have verified that $L$ in (52) when used in the Euler-Lagrange equation reproduces (49) if $w=$ $\frac{2}{3}$. Our final result for the required Lagrangian reads

$$
L=\dot{x} \dot{y}+\frac{\delta}{2}(x \dot{y}-y \dot{x})-\alpha x y+\beta x^{2} y .
$$

In [35] Almendral and Sanjuan made use of Lie theory of differential equations to study the symmetries and integrability of (49) and found that only under certain conditions on the parameters one can obtain the time-dependent first integral of the equation. In particular, for $\alpha=6 \delta^{2} / 2$ such a first integral could be written as

$$
K(x, \dot{x}, t)=\left[\left(\dot{x}+\frac{2 \delta}{5} x\right)^{2}-\beta x^{3}\right] \mathrm{e}^{\frac{6}{5} \delta t} .
$$

Also by defining the canonical variables, i.e.,

$$
p=\sqrt{2}\left(\dot{x}+\frac{2 \delta}{5} x\right) \mathrm{e}^{\frac{3}{5} \delta t} \text { and } q=\sqrt{2} x \mathrm{e}^{\frac{2}{5} \delta t},
$$

it was possible to construct a Hamiltonian function

$$
H(q, p, t)=\frac{1}{2}\left(p^{2}-\frac{\sqrt{2} \beta}{3} q^{3}\right) \mathrm{e}^{-\delta t / 5}
$$

which via the Hamilton's equations leads to the Helmholtz equation for the chosen value of $\alpha$. It is straightforward to use the transformation

$$
L(q, \dot{q}, t)=(p \dot{q}-L(q, \dot{q}, t))_{p=p(q, \dot{q}, t)}
$$

to obtain a time-dependent Lagrangian function

$$
L=\mathrm{e}^{\delta t}\left(\dot{x}^{2}+\frac{4 \delta}{5} x \dot{x}+\frac{4 \delta^{2}}{25} x^{2}+\frac{2 \beta}{3} x^{3}\right)
$$

for the Helmholtz equation under the given parametric condition. 
(vi) The Van der Pol equation

$$
\ddot{x}-\mu\left(1-x^{2}\right) \dot{x}+x=0
$$

for a positive parameter $\mu$, it represents an oscillator which absorbs energy from the surrounding area when $x<1$ and dissipates energy when $x>1$. Periodic motion of this type is qualified as relaxation oscillations. A great variety of physical processes, ranging from the economic crisis to the beating of the human heart, can be modeled by (59). Following the method used for the Helmholtz oscillator, we found the Lagrangian

$$
L=\dot{x} \dot{y}+\frac{\mu}{2}(\dot{x} y-\dot{y} x)+\frac{\mu}{4}\left(x^{3} \dot{y}-x^{2} y \dot{x}\right)-x y
$$

which can reproduce both (59) and its associate equation given by

$$
\ddot{y}+\mu\left(1-x^{2}\right) \dot{y}+y=0 \text {. }
$$

\section{Hamiltonizing non-standard Lagrangians}

We shall present here the Hamiltonian formulation of classical mechanics for nonlinear systems treated in Sect. 3. The Hamiltonian mechanics provides a framework for the theoretical extension to other advanced areas of physics including quantum mechanics [47]. For historical reasons, we shall begin with the so-called Jacobi integral which provides a statement for the conservation of energy [20]. For a first-order Lagrangian $L\left(\dot{q}_{i}, q_{i}, t\right)$ involving only the generalized coordinates and velocities, the Jacobi integral

$$
J^{(1)}=\dot{q}_{i} \frac{\partial L}{\partial \dot{q}_{i}}-L
$$

can be found in any standard text book. Admittedly, $J^{(1)}$ represents one of the first integrals of motion and/or is a conserved quantity only if the Lagrangian is not an explicit function of time.

It is not easy to obtain a result similar to that in (62) for the $n$-th order Lagrangian which satisfies (22). However, in the following we provide a simple derivation for the Jacobi integral for a secondorder Lagrangian $L\left(q_{i}, \dot{q}_{i}, \ddot{q}_{i}, t\right)$ satisfying the differential equation

$$
\frac{\mathrm{d}^{2}}{\mathrm{~d} t^{2}}\left(\frac{\partial L}{\partial \ddot{q}_{i}}\right)-\frac{\mathrm{d}}{\mathrm{d} t}\left(\frac{\partial L}{\partial \dot{q}_{i}}\right)+\frac{\partial L}{\partial q_{i}}=0 .
$$

The total time derivative of this Lagrangian can be written as

$$
\frac{\mathrm{d} L}{\mathrm{~d} t}=\dot{q}_{i} \frac{\partial L}{\partial q_{i}}+\ddot{q}_{i} \frac{\partial L}{\partial \dot{q}_{i}}+\dddot{q}_{i} \frac{\partial L}{\partial \ddot{q}_{i}}+\frac{\partial L}{\partial t} .
$$

If we now substitute $\frac{\partial L}{\partial q_{i}}$ obtained from (63) in (64) and, subtract and add $\ddot{q}_{i} \frac{\partial L}{\partial \dot{q}_{i}}$ to the resulting expression, we arrive at

$$
\frac{\mathrm{d} L}{\mathrm{~d} t}=\frac{\mathrm{d}}{\mathrm{d} t}\left[\ddot{q}_{i} \frac{\partial L}{\partial \ddot{q}_{i}}+\dot{q}_{i} \frac{\partial L}{\partial \dot{q}_{i}}-\dot{q}_{i} \frac{\mathrm{d}}{\mathrm{d} t}\left(\frac{\partial L}{\partial \ddot{q}_{i}}\right)\right]+\frac{\partial L}{\partial t},
$$

so as to identify

$$
J^{(2)}=\ddot{q}_{i} \frac{\partial L}{\partial \ddot{q}_{i}}+\dot{q}_{i} \frac{\partial L}{\partial \dot{q}_{i}}-\dot{q}_{i} \frac{\mathrm{d}}{\mathrm{d} t} \frac{\partial L}{\partial \ddot{q}_{i}}-L
$$

as the Jacobi integral of the second-order Lagrangian $L\left(q_{i}, \dot{q}_{i}, \ddot{q}_{i}, t\right)$. It is straightforward to verify that for the cubic-quintic Duffing oscillator the Jacobi integrals $J^{(1)}$ 's computed by using the Lagrangians in (29) and (30) are in exact agreement with the constant of the motion $K(x, \dot{x})$ given in $(28)$ and $1 / K(x, \dot{x})$. Similar conclusions also hold good for the Lagrangians of the Liénard-type oscillator and Mathews-Lakshmanan oscillator.

For dissipative systems, we first calculate the Jacobi integral for the damped harmonic oscillator by using (62) and the Lagrangian function in (25) and find

$$
J_{d h o}^{(1)}=\dot{x} \dot{y}+\omega^{2} x y .
$$

The result in (67) does not have any effect of dissipation and is exactly the same as that found for two uncoupled harmonic oscillators using their indirect analytic or Lagrangian representation [48]. The reason for this is that the damped harmonic and its associate form a conservative system. However, the time derivative of (67) can be written in the form

$$
\frac{\mathrm{d} J_{d h o}^{(1)}}{\mathrm{d} t}=\dot{y}\left(\ddot{x}+\gamma \dot{x}+\omega^{2} x\right)+\dot{x}\left(\ddot{y}-\gamma \dot{y}+\omega^{2} y\right)
$$

Since $\frac{\mathrm{d} J_{d h_{0}}^{(1)}}{\mathrm{d} t}$ can be made to vanish by using the equations of motion for the damped harmonic oscillator and its associate, the expression in (68) really represents a constant of the motion for the Bateman dual system. The Jacobi integrals for the Lorentz and Van der Pol oscillators are given by

$$
J_{L}^{(1)}=\dot{x} \dot{y}+\alpha x^{2} y-\mu x y
$$

and

$$
J_{V P}^{(1)}=\left.J_{d h o}^{(1)}\right|_{\omega=1}
$$

respectively.

The Abraham-Lorentz system is described by a second-order Lagrangian given in (48). Therefore, its Jacobi integral will be computed using (66). From (48), we have

$$
\frac{\partial L}{\partial \ddot{x}}=-\frac{m \tau}{2} \dot{y}, \quad \frac{\partial L}{\partial \ddot{y}}=\frac{m \tau}{2} \dot{x}
$$

and

$$
\frac{\partial L}{\partial \dot{x}}=m \dot{y}+\frac{m \tau}{2} \ddot{y}, \quad \frac{\partial L}{\partial \dot{y}}=m \dot{x}-\frac{m \tau}{2} \ddot{x} .
$$

From (66), (71) and (72), we get the Jacobi integral for the Abraham-Lorentz equation as

$$
J_{A L}^{(2)}=m \dot{x} \dot{y}+m \tau(\dot{x} \ddot{y}-\dot{y} \ddot{x})+k x y .
$$

It is straightforward to see for the first-order Lagrangian, the Jacobi integral in (62) provides a useful basis for a smooth transition from the description of mechanical systems in $\left(q_{i}, \dot{q}_{i}\right)$ space to that in $\left(q_{i}, p_{i}\right)$ space. This can be achieved by first introducing the definition of canonical momentum 


$$
p_{i}=\frac{\partial L}{\partial \dot{q}_{i}}
$$

and then replacing the Jacobi integral by the Hamiltonian function $H$ to write

$$
H(q, p, t)=\left.\left\{p_{i} \dot{q}_{i}-L(q, p, t)\right\}\right|_{\dot{q}=\dot{q}(q, \dot{q}, t)} .
$$

It is well known that Euler-Lagrange equations result from Hamilton's principle considered in the $(q, \dot{q}, t)$ space. Similarly, a variational principle in the phase space [49] leads to Hamilton's equations of motion

$$
\dot{q}_{i}=\frac{\partial H}{\partial p_{i}} \quad \text { and } \quad \dot{p}_{i}=-\frac{\partial H}{\partial q_{i}} .
$$

The recipe given above for going from Lagrangian to Hamiltonian holds good for the standard Lagrangian. It is of interest to verify if (74) and (75) are also true for non-standard Lagrangians. To that end, we make use of (74) and (75) to obtain the Hamiltonian function

$$
H=\frac{p^{2}}{24}+6 a x^{2}+3 b x^{4}+2 c x^{6}
$$

for the Lagrangian in (29) of the cubic-quintic Duffing oscillator. It is easy to combine (76) and (77) to obtain differential equation (25) and thus verify that (77) indeed represent the correct Hamiltonian. A more interesting example in respect of this is provided by the Liénard-type oscillator in (32) for which we wrote two Lagrangian functions, namely, the logarithmic and inverse-type ones. The logarithmic result in (35) when used in the definition of canonical momentum leads to an expression that does not permit us to express $\dot{x}$ as a function of $p$ and $x$ and is therefore unsuitable to give a Hamiltonian representation of the oscillator. Fortunately, this is not the case with the inverse-type Lagrangian of (36). Here the use of (74) gives

$$
\dot{x}=\frac{\sqrt{3 k}-\left(9 \lambda+k^{2} x^{2}\right) \sqrt{p}}{3 k \sqrt{p}}
$$

which in conjunction with (75) leads to the Hamiltonian

$$
H=2 \sqrt{\frac{p}{3 k}}-\frac{p}{k}\left(\frac{1}{3} k^{2} x^{2}+3 \lambda\right) .
$$

Results similar to those in (78) and (79) for the Lagangian in (39) of the Mathews-Lakshmanan oscillator read

$$
\dot{x}=\frac{p}{2 \lambda}\left(1+\lambda x^{2}\right)
$$

and

$$
H=\frac{p^{2}}{4 \lambda}\left(1+\lambda x^{2}\right)-\frac{\omega^{2}}{\left(1+\lambda x^{2}\right)^{2}} .
$$

The other Lagrangian in (40) is not suitable to provide a similar Hamiltonian representation of the system.

We have found indirect Lagrangian representations for the damped harmonic oscillator, Helmholtz and Van der Pol oscillators. These systems can also be hamiltonized by using the procedure followed above. For example, the well known results for canonical momenta and Hamiltonian of the damped harmonic oscillator are given by [10]

$$
p_{x}=\dot{y}-\frac{\gamma}{2} y, \quad p_{y}=\dot{x}+\frac{\gamma}{2} x,
$$

and

$$
H=p_{x} p_{y}+\frac{\gamma}{2}\left(y p_{y}-x p_{x}\right)+\left(1-\frac{\gamma^{2}}{4}\right) x y .
$$

Here $p_{x}$ and $p_{y}$ stand for canonical conjugate momenta corresponding to $x$ and $y$ coordinates. The results for the canonical momenta and Hamiltonian for the Helmholtz oscillator closely resemble those of the damped harmonic oscillator and are given by

$$
p_{x}=\dot{y}-\frac{\delta}{2} y, \quad p_{y}=\dot{x}+\frac{\delta}{2} x
$$

and

$H=p_{x} p_{y}+\frac{\delta}{2}\left(y p_{y}-x p_{x}\right)-\beta x^{2} y+\left(\alpha-\frac{\delta^{2}}{4}\right) x y$.

Similar results for the Van der Pol oscillator read

$$
\begin{aligned}
& p_{x}=\dot{y}-\frac{\alpha}{2} y+\frac{\alpha}{4} x^{2} y, \\
& p_{y}=\dot{x}+\frac{\alpha}{2} x-\frac{\alpha}{4} x^{3},
\end{aligned}
$$

and

$$
\begin{aligned}
H & =p_{x} p_{y}-\frac{\alpha}{2}\left(x p_{x}-y p_{y}\right)-\frac{\alpha}{4} x^{2}\left(y p_{y}-x p_{x}\right) \\
& +\frac{\alpha^{2}}{4} x^{3} y\left(1-\frac{1}{4} x^{2}\right) y+\left(1-4 \alpha^{2}\right) x y .
\end{aligned}
$$

The Abraham-Lorentz equation (41) is characterized by a second-order Lagrangian. Consequently, it was hamiltonized by Englert [37] by applying Ostrogradsky formalism [50] to generalized momenta. More recently Bender et al. [51] found a simpler quadratic Hamiltonian for the system.

\section{Concluding remarks}

The inverse problem for systems described by linear differential equation has an old root in the classical mechanic's literature [52]. This is, however, not true for nonlinear equations. Only in the recent past, there were attempts $[13,15,16]$ to preserve the Lagrangian structure of nonlinear differential equations. The nonlinear systems were found to admit non-standard Lagrangian representation. These Lagrangians were proposed to identify the class of equations that admit analytic description. For example, in [13] a general form of (5) written as $L=1 /(\dot{x}+k U(x, t))$ was substituted in the Euler-Lagrange equation to verify that this proposed expression of $L$ stands for the Lagrangian for the second-order Riccati equation provided we choose $U=c_{0}(t)+c_{1}(t) x+c_{2}(t) x^{2}$. In this work we have chosen to work with two different approaches to obtain both standard and nonstandard Lagrangian representations of nonlinear systems; (i) For velocity-independent equations we employed (13) to construct the Lagrangian functions from their first integrals and (ii) we adapted the method followed in [22] to obtain similar results for velocity-dependent equations. 
The method used in (i) clearly demonstrates how one dimensional autonomous dynamical systems can have infinitely many analytic representations corresponding to different functions of the first integral. In this context, the results for the non-standard Lagrangians in (30), (36) and (40) obtained by using reciprocal of the first integrals can be regarded as a useful addendum to those found in the existing literature $[15,16]$. One may, however, try to improve on our approach by introducing more efficient methods to compute first integrals. Such studies, on the one hand, are likely to broaden the scope of applicability of our approach and, on the other hand, are likely to shed new light on the system integrability [53]. The indirect analytic representation given in (3) for the damped harmonic oscillator is a very well-known result in the classical mechanic's literature. But it appears that we do not have similar results for equations of nonlinear dissipative systems. Thus we made use of the method in (ii) to obtain the Lagrangian functions in (48), (53) and (60) for three physically important dissipative nonlinear systems. In this context, we also computed results for Jacobi integrals to provide appropriate Hamiltonian representations of the systems.

The inverse problems in natural sciences, ranging from geophysics to medical diagnostics, have been widely discussed in the literature [54]. Here one attempts to discover the cause of a set of observed data. In classical mechanics, however, the solution of the inverse problem consists in expressing an ordinary differential equation (linear or nonlinear) in Hamilton's variational form. It remains an interesting curiosity to extend our treatment to field theory [55] where the time evolution of physical systems is expressed by partial differential equations.

\section{Appendix: Lagrangian and Jacobi integral of (26) with variable coefficients}

We consider the equation of motion of the oscillator written as

$$
\ddot{x}+\gamma \dot{x}+a(t) x+b(t) x^{3}+c(t) x^{5}=0 .
$$

Here the system is not acted on by an external force and the damping coefficient $\gamma$ is independent of time. As regards the inverse problem of the calculus of variation, the external force $F(t)$ always contributes a term $q(t) F(t)$ to the Lagrangian function [48] and in all practical situations, the damping coefficient does not depend on time. Following our treatment of the Helmholtz oscillator we introduce

$$
\bar{L}=y(t)\left(\ddot{x}+\gamma \dot{x}+a(t) x+\omega_{1} b(t) x^{3}+\omega_{2} c(t) x^{5}\right) .
$$

Here $\omega_{1}$ and $\omega_{2}$ represent weight factors that we need to obtain the correct Bateman-type Lagrangian for the nonlinear equation (90). The second-order Lagrangian in the $x$ variable gives the associated equation

$$
\ddot{y}-\gamma \dot{y}+a(t) y+3 \omega_{1} b x^{2} y+5 \omega_{2} c(t) x^{4} y=0 .
$$

For the final solution of the inverse problem we now introduce

$$
\begin{aligned}
L & =\bar{L}+x\left(\ddot{y}-\gamma \dot{y}+a(t) y+3 \omega_{1} b(t) x^{2} y\right. \\
& \left.+5 \omega_{2} c(t) x^{4} y\right)-\frac{\mathrm{d}}{\mathrm{d} t}(\dot{x} y+\dot{y} x),
\end{aligned}
$$

which via the Euler-Lagrange equation

$$
\frac{\mathrm{d}}{\mathrm{d} t}\left(\frac{\partial L}{\partial \dot{y}}\right)-\frac{\partial L}{\partial y}=0
$$

yields the oscillator equation (90) provided $\omega_{1}=\frac{1}{2}$ and $\omega_{2}=\frac{1}{3}$. Thus we have

$L=\dot{x} \dot{y}+\frac{x \dot{y}-y \dot{x}}{2}-a(t) x y-b(t) x^{3} y-c(t) x^{5} y$.

The Lagrangian in (95) gives the associated equation (equation for the $y$ degree of freedom) and we have

$$
\ddot{y}-\lambda \dot{y}+a(t) y+3 b(t) x^{2} y+5 c(t) x^{4} y=0 .
$$

It is easy to verify that for $\gamma=0$ the Lagrangian in (94) provides an indirect analytic representation for (26) with time-dependent coefficients.

For $\gamma=0$ the Lagrangian in (25) gives an indirect analytic representation of two uncoupled harmonic oscillators. The standard harmonic oscillator Lagrangian can be found from it using the prescription $y, \dot{y} \rightarrow x, x$. This approach, however, needs some modification for application to nonlinear systems. For the undamped cubic-quintic oscillator we first modify (94) to read

$$
\begin{aligned}
L_{0} & =L(\gamma=0, y, \dot{y}=x, x)=\frac{1}{2} \dot{x}^{2}-\frac{1}{2} a(t) x^{2} \\
& -\frac{1}{2} \omega_{3} b(t) x^{4}-\frac{1}{2} c(t) x^{6}
\end{aligned}
$$

and then demand that the Euler-Lagrange equation for $L_{0}$ should reproduce (26) with time-dependent coefficients. In this way we get $\omega_{3}=\frac{1}{2}$ and $\omega_{4}=\frac{1}{3}$ such that $L_{0}$ has the same form as the result in (29).

Substituting (96) in (57) the Jacobi integral $J_{0}^{(1)}$ for the undamped oscillator obtained from (90) comes out as

$$
J_{0}^{(1)}=\frac{1}{2} \dot{x}^{2}+\frac{1}{2} a(t) x^{2}+\frac{1}{4} b(t) x^{4}+\frac{1}{6} c(t) x^{6} .
$$

The time derivative of (97) tells us that the Jacobi integral $J_{0}^{(1)}$ is a constant of the motion under the constraint

$$
\frac{1}{2} \dot{a}(t) x^{2}+\frac{1}{4} \dot{b}(t) x^{4}+\frac{1}{6} \dot{c}(t) x^{6}=0 .
$$

Clearly, for time-independent coefficients, the constraint disappears such that the related Jacobi integral is now equal to the constant of the motion $K(x, \dot{x})(28)$ to within a multiplicative constant.

The Lagrangian (94) having two degrees of freedom leads to the Jacobi integral

$$
J_{\gamma}^{(2)}=\dot{x} \dot{y}+a(t) x y+b(t) x^{3} y+c(t) x^{5} y
$$


for the undamped system. In view of (89) and (95) it can be shown $J_{\gamma}^{(2)}$ is a constant of the motion provided

$$
\dot{a}(t) x y+\dot{b}(t) x^{3} y+\dot{c}(t) x^{5} y=0
$$

which again disappears when coefficients of (89) are time independent.

\section{References}

[1] R.M. Santilli, Foundation of Theoretical Mechanics: The inverse Problem in Newtonian Mechanics, Vol. 1 Springer Verlag, New York, 1978.

[2] H. Helmholtz, J. Reine Angew. Math. 100, 137 (1887).

[3] F. Pardo, J. Math. Phys. 30, 2045 (1989).

[4] P. Caldirola, Il Nuovo Cimento 18, 393 (1941).

[5] E. Kanai, Prog. Theor. Phys. 3, 440 (1948).

[6] M.C. Baldiotti, R. Fresneda, D.M. Gitman, Phys. Lett. A 375, 1630 (2011).

[7] H. Bateman, Phys. Rev. 38, 815 (1931).

[8] P.M. Morse, H. Feshbach, Methods of Theoretical Physics, Part I, McGraw-Hill Education, New York 1953.

[9] H. Goldstein, Classical Mechanics, Addison-Wesley, Reading (MA) 1950.

[10] M. Blasone, P. Jizba, Ann. Phys. (NY) 312, 354 (2004).

[11] K. Takahashi, J. Math. Phys. 59, 032103 (2018)

[12] K. Takahashi, J. Math. Phys. 59, 072108 (2018).

[13] J.F. Cariñena, M.F. Rañada, F. Santander, J. Math. Phys. 46, 062703 (2005).

[14] V.K. Chandrasekar, M. Senthivelan, M. Lakshmanan, J. Math. Phys. 48, 032701 (2007).

[15] Z.E. Musielak, J. Phys. A Math. Theor. 41, 055205 (2008).

[16] J.L. Cieśliński, T. Nikiciuk, J. Phys. A Math. Theor. 43, 175205 (2010).

[17] E.L. Ince, Ordinary Differential Equations, Dover Publications, New York 1958.

[18] A. Saha, B. Talukdar, Rep. Math. Phys. 73, 299 (2014).

[19] M.C. Nucci, K.M. Tamizhmani, J. Nonlin. Math. Phys. 17, 167 (2010).

[20] E.T. Whittaker, A Treatise on the Analytical Dynamics of Particles and Rigid Bodies, Cambridge University Press, Cambridge 1988, first published 1904 .

[21] G. López, Ann. Phys. (NY) 251, 363 (1996).

[22] S.A. Hojman, J. Phys. A: Math. Gen. 17, 2399 (1984).
[23] P.J. Olver, Application of Lie Groups to Differential Equations, 1st ed. Springer, New York 1993.

[24] I.N. Sneddon, Elements of Partial Differential Equations, McGraw-Hill, New York, 1957.

[25] S. Ghosh, J. Shamanna, B. Talukdar, Can. J. Phys. 82, 561 (2004).

[26] G. Morandi, C. Ferrario, G.L. Vecchio, G. Marmo, C. Rubano, Phys. Rep. 188, 147 (1990).

[27] R. Hojman, S.A. Hojman, J. Sheinbaum, Phys. Rev. D 28, 1333 (1983).

[28] D.G. Currie, E.J. Saletan, J. Math. Phys. 7, 967 (1966)

[29] R. Courant, D. Hilbert, Methods of Mathematical Physics, Vol. 1, Wiley Eastern, New Delhi 1975.

[30] B. Talukdar, U. Das, Higher-Order Systems in Classical Mechanics, Norasa Publishing House, New Delhi, 2008.

[31] C. Caratheodory, Calculus of Variations and Partial Differential Equations of First Order, Vol. 2, 2nd ed. Holden Day, San Fransisco 1967.

[32] A. Elías-Zúñiga, App. Math. Modelling 37, 2574 (2013).

[33] P.M. Mathews, M. Lakshmanan, Il Nuovo Cimento A 26, 299 (1975).

[34] J.D. Jackson, Classical Electrodynamics (reprint), Nice Printing press, Delhi 2011.

[35] J.A. Almendral, M.A. F. Sanjuán, J. Phys. A: Math. Gen. 36, 695 (2003).

[36] B. Van der Pol, J. Van der Mark, Nature 120, 363 (1927).

[37] B. Englert, Ann. Phys. (NY) 129, 1 (1980).

[38] A.H. Nayfeh and D.T. Mook, Nonlinear oscillations, John Wiley, New York 1995.

[39] E. Urenda-Cázares, A. Gallegos, J.E. Macías-Díaz, H. Vargas-Rodríguez, Commun. Nonlinear Sci. Numer. Simul. 78, 104860 (2019).

[40] N.H. Ibragimov, Elementary Lie Group Analysis and Ordinary Differential Equations, John Wiley and Sons, Chichester (UK) 1999.

[41] V.K. Chandrasekar, M. Senthilvelan, M. Lakshmanan, Phys. Rev. E 72, 066203 (2005).

[42] R. Delbourgo, A. Salam, J. Strathdee, Phys. Rev. 187, 1999 (1969).

[43] R. Koc, M. Koca, J. Phys. A: Math. Gen. 36, 8105 (2003).

[44] M. Lakshmanan, V.K. Chandrasekar, Eur. Phys. J. Spec. Top. 222, 665 (2013).

[45] Z. Feng, G. Gao, J. Cui, Comm. Pure Appl. Anal. 10, 1377 (2011). 
[46] S. Ghosh, B. Talukdar, P. Sarkar, U. Das, Acta Mech. 190, 73 (2007).

[47] L.I. Schiff, Quantum Mechanics, Tata McGraw-Hill Edition, New Delhi 2010.

[48] B. Talukdar, S. Chatterjee, S.G. Ali, Pramana J. Phys. 94, 141 (2020).

[49] J. Shamanna, B. Talukdar, U. Das, Phys. Lett. A 305, 93 (2002).

[50] M. Ostrogradsky, Mem. Ac. St. Petersbourg VI, 385 (1850).

[51] C.M. Bender, M. Gianfreda, N. Hassanpour, H.F. Jones, J. Math. Phys. 57, 084101 (2016).
[52] J. Douglas, Trans. Amer. Math. Soc. 50, 71 (1941).

[53] T. Stachowiak, J. Diff. Equations, 266, 5895 (2019).

[54] Ill-Posed Problems in the Natural Sciences, Mathematics and Mechanics Series, Eds. A.N. Tikhonov, A.V. Goncharsky, Translated by B.M. Bloch, MIR Publisher, Moscow 1987.

[55] L.E. Malvern, Introduction to the Mechanics of a Continuous Medium, Prentice-Hall, New Jersey 1969. 